\title{
Theoretical Overview on Recent Developments in Transverse Spin Physics
}

\author{
Feng Yuan \\ RIKEN/BNL Research Center, Building 510A, Brookhaven National Laboratory, Upton, NY 11973 \\ Nuclear Science Division, Lawrence Berkeley National Laboratory, Berkeley, CA 94720, USA ${ }^{1}$
}

\begin{abstract}
Transverse-spin physics has been very active and rapidly developing in the last few years. In this talk, I will briefly summarize recent theoretical developments, focusing on the associated QCD dynamics in transverse spin physics.
\end{abstract}

Keywords: Single transverse-spin asymmetries

PACS: $12.38 . \mathrm{Bx}, 12.39 . \mathrm{St}, 13.85 . \mathrm{Qk}$

There have been strong experimental interests on transverse spin physics around the world, from the deep inelastic scattering experiments such as the HERMES collaboration at DESY, SMC at CERN, and Hall A and CLAS at JLab, the proton-proton collider experiment from RHIC at Brookhaven, and the very relevant $e^{+} e^{-}$annihilation experiment from BELLE at KEK. One of the major goals in transverse spin physics is to study the quark transversity distribution, the last unknown leading-twist quark distribution in nucleon. Besides the quark transversity distribution, the transverse spin physics also opened a new window to explore the partonic structure of nucleon, the so-called transverse momentum dependent (TMD) parton distributions. TMD parton distribution is an extension to the usual Feynman parton distributions. These distributions allow us to study the three-dimension picture of partons inside the nucleon, and they are also closely related to the generalized parton distributions and the parton orbital angular momenta. Especially, the single transverse spin asymmetry (SSA) phenomena in high energy hadronic processes have attracted many theoretical and experimental investigations. The SSA is defined as the asymmetry when one of the hadrons' transverse spin is flipped, $A_{N} \sim\left(d \sigma\left(S_{\perp}\right)-d \sigma\left(-S_{\perp}\right)\right) /\left(d \sigma\left(S_{\perp}\right)-d \sigma\left(-S_{\perp}\right)\right)$. It has been a great theoretical challenge in the understanding of these phenomena. This is because the leading partonic contribution to the SSA vanish in the leading order, whereas the experimental observation show that these SSAs are in tens of percentage in the forward scattering of the polarized nucleon.

Recent theoretical developments have made great progress in the exploration of the underlying physics for the single spin phenomena. It is impossible to cover all these exciting physics in this short talk. Rather, I would like to focus on one important subject,

\footnotetext{
${ }^{1}$ This work was supported in part by the U.S. Department of Energy under contract DE-AC0205CH11231. We are grateful to RIKEN, Brookhaven National Laboratory and the U.S. Department of Energy (contract number DE-AC02-98CH10886) for providing the facilities essential for the completion of this work.
} 
i.e., the nontrivial QCD dynamics associated with transverse spin physics: the QCD factorization, the universality of the parton distributions and fragmentation functions, and their scale evolutions.

Among those TMD parton distributions and fragmentation functions, two functions have been mostly discussed: the Sivers quark distribution and the Collins fragmentation function. The Sivers quark distribution represents a distribution of unpolarized quarks in a transversely polarized nucleon, through a correlation between the quark's transverse momentum and the nucleon polarization vector. The Collins function represents a correlation between the transverse spin of the fragmenting quark and the transverse momentum of the hadron relative to the "jet axis" in the fragmentation process. Although they both belong to the so-called "naive-time-reversal-odd" functions, they do have different universality properties. For the quark Sivers function, because of the initial/final state interaction difference, they differ by signs for the SIDIS and Drell-Yan processes $[1,2,3,4]$. On the other hand, there have been several studies showing that the Collins function is universal between different processes, primarily in the SIDIS and $e^{+} e^{-}$annihilation $[5,6,7,8]$, and recently in $p p$ collisions [9]. In the following, I will take the example of the Collins contribution to the azimuthal asymmetric distribution of hadrons inside a high energy jet in the transversely polarized $p p$ collision to demonstrate this universality property,

$$
p\left(P_{A}, S_{\perp}\right)+p\left(P_{B}\right) \rightarrow \operatorname{jet}\left(P_{J}\right)+X \rightarrow H\left(P_{h}\right)+X,
$$

where a transversely polarized proton with momentum $P_{A}$ scatters on another proton with momentum $P_{B}$, and produces a jet with momentum $P_{J}$. The three momenta of $P_{A}$, $P_{B}$ and $P_{J}$ form the so-called reaction plane. Inside the produced jet, the hadrons are distributed around the jet axis, where we define transverse momentum $P_{h T}$ relative to the jet axis. The correlation between $P_{h T}$ and the polarization vector $S_{\perp}$ introduces the Collins contribution to the single spin asymmetry in this process.

We need to generate a phase from the scattering amplitudes to have a non-vanishing SSA. If the phase comes from the vertex associated with the fragmenting quark and the final state hadron, or from the dressed quark propagator, it is easy to argue the universality of the Collins function between this process and the SIDIS $/ e^{+} e^{-}$process, because they are the same. The main issue of the universality discussion concerns the extra gluon exchange contribution between the spectator of the fragmentation process and hard partonic part. In Fig. 2, we have shown all these interactions for a particular partonic channel $q q^{\prime} \rightarrow q q^{\prime}$ contribution, including the gluon attachments to the incident quarks $(\mathrm{a}, \mathrm{c})$, and final state balancing quark (d) and the internal gluon propagator (b). The contributing phases of the diagrams in Fig. 2 come from the cuts through the internal propagators in the partonic scattering amplitudes. In Fig. 2, we labeled these cut-poles by short bars in the diagrams. From the calculations, we will find that all these poles come from a cut through the exchanged gluon and the fragmenting quark in each diagram, and all other contributions either vanish or cancel out each other. For example, in Fig. 2(d), we show two additional cuts, which contribute however opposite to each other and cancel out completely. Therefore, by using the Ward identity at this particular order, the final results for all these diagrams will sum up together into a factorized form, where the cross section is written as the hard partonic cross section for $q\left(S_{\perp}\right) q^{\prime} \rightarrow q\left(s_{\perp}\right) q^{\prime}$ subprocess 


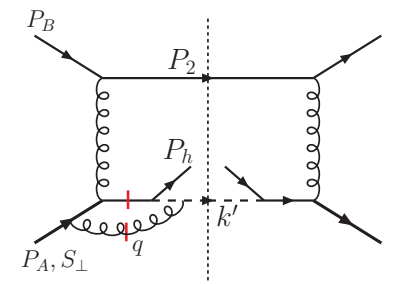

(a)

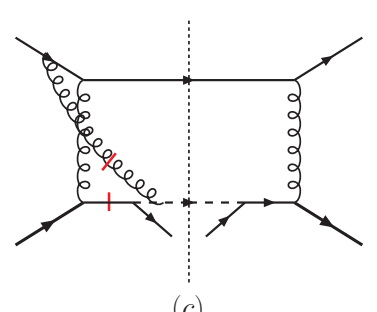

(c)

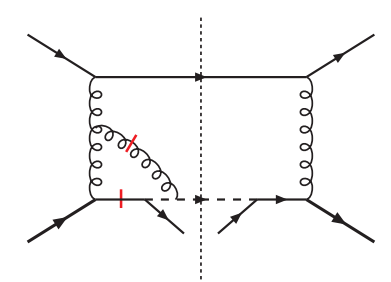

(b)

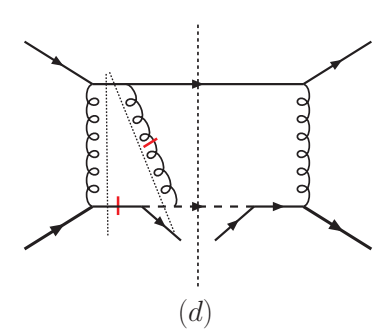

FIGURE 1. Gluon exchange diagrams contributions to the Collins asymmetry in pp collisions. The short bars indicate the pole contributions to the phase needed for a non-vanishing SSA. The additional two cuts in (d) cancel out each other.

multiplied by a Collins fragmentation function. The exchanged gluon in Fig. 2 is now attaching to a gauge link from the fragmentation function definition. Similar calculations can be performed for the other two processes SIDIS and $e^{+} e^{-}$annihilation, and the same Collins function will be observed. This argument can also be extended to two-gluon exchange diagrams [9].

The key steps in the above derivation are the eikonal approximation and the Ward identity. The eikonal approximation is valid when we calculate the leading power contributions in the limit of $P_{h T} \ll P_{J}$. The Ward identity ensure that when we sum up the diagrams with all possible gluon attachments we shall get the eikonal propagator from the gauge link in the definition of the fragmentation function. The most important point to apply the Ward identity in the above analysis is that the eikonal propagator does not contribute to the phase needed to generate a nonzero SSA.

This observation is very different from the SSAs associated with the parton distributions, where the eikonal propagators from the gauge link in the parton distribution definition play very important role [1, 2, 3, 4]. It is the pole of these eikonal propagators that contribute to the phase needed for a nonzero SSA associated with the naive-timereversal-odd parton distributions, which also predicts a sign difference for the quark Sivers function between the SIDIS and Drell-Yan processes. More complicated results have been found for the SSAs in the hadronic dijet-correlation [10,11], where a normal TMD factorization breaks down [12]. The reason is that the eikonal propagators from the initial and final state interactions in dijet-correlation process do contribute poles in the cross section [11, 12]. Because of this, the Ward identity is not applicable, and the standard TMD factorization breaks down, although a modified factorization may be valid if we modify the definition of the TMD parton distributions to take into account all the initial and final state interaction effects [10]. 
In particular, there is a sign change between the SSAs in SIDIS and Drell-Yan processes $[1,2]$,

$$
\text { Sivers SSA }\left.\right|_{\mathrm{DY}}=- \text { Sivers SSA }\left.\right|_{\text {DIS }} \text {. }
$$

This nontrivial result of the opposite signs between the above two processes will still hold when gluon radiation contributions are taken into account, where the large transverse momentum Sivers function is generated from the twist-three quark-gluon correlation function [13]. It is of crucial to test this nontrivial QCD predictions by comparing the SSAs in these two processes. The Sivers single spin asymmetry in SIDIS process has been observed by the HERMES collaboration, and the planned Drell-Yan measurement at RHIC and other facility will test this prediction.

Another interesting probe for the initial/final state interaction effects is the SSA in heavy quark and antiquark production in hadronic process. Because the heavy quark and antiquark can be detected by their decay products, their SSAs can be measured separately. The heavy quark and antiquark produced in short distance partonic processes will experience different final state interactions with the nucleon spectator due to their different color charges, and therefore the SSAs for heavy quark and antiquark will be different. Detailed calculations show that the difference could be as large as a factor of 3 if the quark-antiquark channel contribution dominates [14]. Certainly, heavy quark production in high energy hadronic process is dominated by gluon-gluon fusion contributions. Therefore, the single spin asymmetry from gluonic sector will be important at RHIC energy. This part of contribution has recently been studied in the twist-three approach [15]. The twist-three three-gluon correlation functions contain two independent functions because of different color factors: one with $f_{a b c}$ and one with $d_{a b c}$ where $a, b, c$ are the color indices for the three gluons [16]. Both correlations and the quark-gluon correlation function mentioned above will contribute to heavy quark single spin asymmetry in $p p$ collisions. However, at RHIC energy kinematics, the quark-gluon correlation functions alone generate only a very small asymmetry for open charm production. Therefore, the observation of any significant single-spin asymmetry would be a clear indication of the presence of three-gluon correlations inside a polarized proton [15].

Most recently, there has been very exciting progress in studying the scale evolution equations for the quark-gluon and three-gluon correlation functions and their implications to the energy dependence of the relevant SSA observables [17, 18, 19]. General structure of the evolution equations for the twist-three quark-gluon correlation functions has been known in the literature [20]. However, the correlation functions responsible to the single transverse spin asymmetries are special projections of the general twistthree quark-gluon correlations, and their evolutions are not directly available from the already known results [20]. Earlier attempts [21] have been made to derive the evolution equations for the correlation functions, but were not complete. On the other hand, from the large transverse momentum quark Sivers function calculated in [13], we would already obtain the evolution equation for $T_{F}(x)$ (which is the transverse momentum moment of the quark Sivers function), since the collinear divergence in that calculation will lead to the splitting function of $T_{F}(x)$. This splitting function was confirmed by a complete calculation of next-to-leading order QCD correction to the transverse-momentum weighted spin asymmetry in Drell-Yan lepton pair production [17] and the derivations of the scale evolution equations directly $[18,19]$. In particular, the scale evolution for 
the quark-gluon correlation function $T_{F}(x)$ is found to be,

$$
\begin{aligned}
\frac{\partial}{\partial \ln \mu^{2}} T_{F}\left(x_{B}, \mu^{2}\right)= & \frac{\alpha_{s}}{2 \pi} \int \frac{d x}{x}\left[C_{F}\left\{\frac{1+z^{2}}{(1-z)_{+}}+\frac{3}{2} \delta(1-z)\right\} T_{F}(x, x)\right. \\
& \left.+\frac{C_{A}}{2}\left\{\frac{1+z}{1-z} T_{F}(x z, x)-\frac{1+z^{2}}{1-z} T_{F}(x, x)+\tilde{T}_{F}(x z, x)\right\}\right],
\end{aligned}
$$

where $z=x_{B} / x$.

Furthermore, the NLO perturbative-QCD correction to the transverse momentum weighted single spin asymmetry in Drell-Yan lepton pair production in hadronic collisions has engaged the transverse spin physics to a more solid theoretical ground [17]. It has been shown that the collinear divergences can be absorbed into the NLO twist-three quark-gluon correlation function of the transversely polarized nucleon and the unpolarized quark distribution of the unpolarized nucleon. This calculation suggests that a general factorization formula exists for the transverse momentum weighted spin-dependent cross section in the Drell-Yan process, in extension of the general factorization arguments given in [22].

One important feature of this result is its behavior near "partonic threshold", that is in the large- $z$ limit of the integrand, corresponding to $\hat{s} \sim Q^{2}$, when the initial partons have "just enough" energy to produce the virtual photon. Setting the scale $\mu=Q$, we have the following structure of the NLO correction in this case:

$\frac{d\left\langle q_{\perp} \Delta \sigma\left(S_{\perp}\right)\right\rangle}{d Q^{2}}=\sigma_{0} \frac{\alpha_{s}}{2 \pi} \int \frac{d x}{x} \frac{d x^{\prime}}{x^{\prime}} T_{F}\left(x, x ; \mu^{2}\right) \bar{q}\left(x^{\prime} ; \mu^{2}\right)\left[4 C_{F}\left(1+z^{2}\right)\left(\frac{\ln (1-z)}{1-z}\right)_{+}\right]$

where we only keep the "double-logarithmic" term which dominates near threshold in the $\overline{\mathrm{MS}}$ scheme. The structure of this expression is identical to that for the spin-averaged $q_{\perp}$-integrated NLO cross section near threshold,

$$
\frac{d \sigma}{d Q^{2}}=\sigma_{0} \frac{\alpha_{s}}{2 \pi} \int \frac{d x}{x} \frac{d x^{\prime}}{x^{\prime}} q\left(x ; \mu^{2}\right) \bar{q}\left(x^{\prime} ; \mu^{2}\right)\left[4 C_{F}\left(1+z^{2}\right)\left(\frac{\ln (1-z)}{1-z}\right)_{+}\right] .
$$

This means that the soft gluon contribution is spin-independent. It contributes in the same way to the spin-averaged and single-spin-dependent cross sections, and will lead to the same soft-gluon threshold resummation effects to these cross sections, at least at the leading double logarithmic level. This observation is very similar to that made for the transverse momentum resummation in the Drell-Yan process [23]. This will likely have the phenomenological consequence that the single-spin asymmetry for the DrellYan process will be quite stable under NLO corrections, in particular when $\tau=Q^{2} / \mathrm{s}$ is large.

In summary, transverse spin physics has attracted much attention and has been rapidly developed in the last few years. In this overview, I could not cover all these important developments, rather I emphasized a few examples, including the universality of the parton distribution and fragmentation functions, and QCD evolution and next-to-leading order corrections to the relevant observables. Fortunately, there are many talks on the 
transverse spin physics in this conference, and I believe that they will present more comprehensive reviews on these exciting developments in this physics. This has shown that the transverse spin physics is playing a very important role in the strong interaction physics for hadronic spin physics. We will learn more about QCD dynamics and nucleon structure from these studies.

\section{REFERENCES}

1. S. J. Brodsky, D. S. Hwang and I. Schmidt, Phys. Lett. B 530, 99 (2002); Nucl. Phys. B 642, 344 (2002).

2. J. C. Collins, Phys. Lett. B 536, 43 (2002).

3. X. Ji and F. Yuan, Phys. Lett. B 543, 66 (2002); A. V. Belitsky, X. Ji and F. Yuan, Nucl. Phys. B 656, 165 (2003).

4. D. Boer, P. J. Mulders and F. Pijlman, Nucl. Phys. B 667, 201 (2003).

5. A. Metz, Phys. Lett. B 549, 139 (2002).

6. J. C. Collins and A. Metz, Phys. Rev. Lett. 93, 252001 (2004).

7. L. P. Gamberg, A. Mukherjee and P. J. Mulders, Phys. Rev. D 77, 114026 (2008).

8. S. Meissner and A. Metz, arXiv:0812.3783 [hep-ph].

9. F. Yuan, Phys. Rev. Lett. 100, 032003 (2008); Phys. Rev. D 77, 074019 (2008).

10. C. J. Bomhof, P. J. Mulders and F. Pijlman, Phys. Lett. B 596, 277 (2004); Eur. Phys. J. C 47, 147 (2006); JHEP 0702, 029 (2007); A. Bacchetta, C. J. Bomhof, P. J. Mulders and F. Pijlman, Phys. Rev. D 72, 034030 (2005); C. J. Bomhof and P. J. Mulders, arXiv:0709.1390 [hep-ph].

11. J. W. Qiu, W. Vogelsang and F. Yuan, Phys. Lett. B 650, 373 (2007); Phys. Rev. D 76, 074029 (2007); W. Vogelsang and F. Yuan, Phys. Rev. D 76, 094013 (2007).

12. J. Collins and J. W. Qiu, Phys. Rev. D 75, 114014 (2007); J. Collins, arXiv:0708.4410 [hep-ph].

13. X. Ji, J. W. Qiu, W. Vogelsang and F. Yuan, Phys. Rev. Lett. 97, 082002 (2006); Phys. Rev. D 73, 094017 (2006); Phys. Lett. B 638, 178 (2006).

14. F. Yuan and J. Zhou, arXiv:0806.1932 [hep-ph].

15. Z. B. Kang and J. W. Qiu, Phys. Rev. D 78, 034005 (2008); Z. B. Kang, J. W. Qiu, W. Vogelsang and F. Yuan, Phys. Rev. D 78, 114013 (2008).

16. X. D. Ji, Phys. Lett. B 289, 137 (1992).

17. W. Vogelsang, F. Yuan, to be submitted.

18. Z. B. Kang and J. W. Qiu, arXiv:0811.3101 [hep-ph].

19. J. Zhou, F. Yuan and Z. T. Liang, arXiv:0812.4484 [hep-ph].

20. See, for example: I. I. Balitsky and V. M. Braun, Nucl. Phys. B 311, 541 (1989); P. G. Ratcliffe, Nucl. Phys. B 264, 493 (1986); X. D. Ji and C. h. Chou, Phys. Rev. D 42, 3637 (1990); Y. Koike and K. Tanaka, Phys. Rev. D 51, 6125 (1995); J. Kodaira, Y. Yasui, K. Tanaka and T. Uematsu, Phys. Lett. B 387, 855 (1996); A. V. Belitsky and D. Mueller, Nucl. Phys. B 503, 279 (1997); A. V. Belitsky, Nucl. Phys. B 558, 259 (1999); A. V. Belitsky, Nucl. Phys. B 574, 407 (2000); A. V. Belitsky, X. D. Ji, W. Lu and J. Osborne, Phys. Rev. D 63, 094012 (2001). I. I. Balitsky, V. M. Braun, Y. Koike and K. Tanaka, Phys. Rev. Lett. 77, 3078 (1996).

21. A. A. Henneman, D. Boer and P. J. Mulders, Nucl. Phys. B 620, 331 (2002).

22. J. W. Qiu and G. Sterman, Nucl. Phys. B 353, 105 (1991); Nucl. Phys. B 353, 137 (1991).

23. A. Idilbi, X. d. Ji, J. P. Ma and F. Yuan, Phys. Rev. D 70, 074021 (2004). 